\title{
Más allá de la socialización y de la sociabilidad: jóvenes y bachillerato en México
}

Eduardo Weiss

\section{Resumen}

El artículo presenta la línea de investigación sobre jóvenes y escuela en México. La línea busca construir un puente entre los estudios sobre alumnos y los estudios sobre jóvenes: los estudiantes son a la vez jóvenes y gran parte de la vida juvenil se desarrolla en la escuela. Se enfoca la doble condición de ser estudiante y de ser joven, pero también la condición de ser adolescente hijo o hija de familia. El artículo da cuenta del desarrollo de la línea de investigación y establece desde sus hallazgos una discusión con dos conceptos: el concepto de socialización en los estudios sobre alumnos y el concepto de sociabilidad en el campo de los estudios sobre jóvenes, a la vez que propone restituir el concepto de subjetivación. Se presenta el enfoque metodológico de esta línea de investigación que combina elementos de la tradición etnográfica con elementos de la tradición hermenéutica. En una primera parte el artículo muestra los hallazgos de las investigaciones sobre el sentido del bachillerato y discute desde ahí los límites del concepto de socialización. En una segunda parte presenta los hallazgos sobre la escuela como espacio juvenil. En este espacio se despliegan procesos de socialización y sociabilidad, pero más allá de lo que comprenden estos conceptos es necesario conceptualizar los procesos de subjetivación. Estos procesos no son solitarios: los jóvenes comparten con compañeros, amigos y novios vivencias y conversan sobre ellas. La conversación es una forma privilegiada de reflexión y convierte las vivencias en experiencias que permiten crecer.

\section{Palabras clave}

Estudiantes - Jóvenes - Subjetivación - Socialización - Sociabilidad.
I- Centro de Investigación y de Estudios Avanzados del Instituto Politécnico Nacional (CINVESTAV), Ciudad de México, DF, México.

Contacto: eweiss@cinvestav.mx 


\section{Beyond socialization and sociability: Youth and high school in Mexico}

Eduardo Weiss

\section{Abstract}

This article presents the line of research on youth and school in Mexico. Such research attempts to build a bridge between studies on students and studies on youth: students are also young people and spend a large part of their life in school. It focuses on the double condition of being a student and being young, as well as on the condition of being an adolescent son or daughter. The article explains the development of this line of research and uses its findings to establish a discussion of two concepts: the concept of socialization in studies on students, and the concept of sociability in the field of studies on youth, while proposing a return to the concept of subjectivization. I present the methodological focus of this line of research, which combines elements from the ethnographic tradition with elements from the hermeneutic tradition. The initial part of the article brings research findings on the meaning of high school and discusses the limits of the concept of socialization. The second part presents the findings on school as a youthful space-a space where processes of socialization and sociability unfold. However, beyond these concepts, the processes of subjectivization must be conceptualized. Such processes are not solitary: Young people share and talk about their experiences with classmates, friends, and girlfriends/boyfriends. Conversation is a privileged form of reflection and converts experiences into opportunities for growth.

\section{Keywords}

Students - Youth - Subjectivization - Socialization - Sociability.

I- Centro de Investigación y de Estudios Avanzados del Instituto Politécnico Nacional (CINVESTAV), Ciudad de México, D.F, México. Contact: eweiss@cinvestav.mx 


\section{Introducción}

La línea de investigación que he desarrollado en los últimos quince años con mis estudiantes de maestría y doctorado se centra en el tema Jóvenes y escuela. Como señala el nombre buscamos enfocar a la vez la doble condición, la de ser estudiante y la de ser joven. Y más recientemente ampliamos nuestra mirada hacia una tercera condición: no sólo son estudiantes y jóvenes/adolescentes, también y en primer lugar, son hijas e hijos de familia. El presente artículo busca dar cuenta del desarrollo de esa línea y establecer desde nuestros hallazgos sobre la vida juvenil en las escuelas una discusión con dos conceptos, el concepto de socialización en el campo de los estudios sobre alumnos y el concepto de sociabilidad en el campo de los estudios sobre jóvenes. Considero necesario agregar el concepto de subjetivación para poder dar cuenta de las vivencias de los estudiantes adolescentes y los procesos de reflexión sobre esas vivencias.

La línea de investigación busca construir un puente entre los estudios sobre alumnos y los estudios sobre jóvenes. Los estudios sobre alumnos enfocan usualmente su procedencia social y las trayectorias escolares, así como el logro de aprendizajes. Los estudios sobre juventud(es) las investigan dentro de ambientes extra-escolares, como agentes de culturas juveniles: punks, grafiteros, cibernéticos, etc. (URTEAGA, 2007). Desde al año 2000 se está abriendo camino la investigación sobre los estudiantes como actores inmersos en diferentes experiencias con sus pares y en prácticas socio-culturales, como señala el estado de conocimiento de Guzmán y Saucedo (2005). A ello contribuyó la presente línea de investigación que encontró que para muchos estudiantes la escuela es sobre todo un espacio de vida juvenil y enfocó la doble condición de estudiantes y jóvenes: los estudiantes son a la vez jóvenes y gran parte de la vida juvenil se desarrolla en la escuela.
Gran parte de los estudios sobre estudiantes y jóvenes parten del concepto de socialización, que implica la integración a los valores y normas adultas por los actores juveniles y estudiantiles. Investigadores estadounidenses señalaron desde los años cincuenta más allá de esta socialización intergeneracional, la importancia de la socialización intra-generacional: los adolescentes y jóvenes tienen un mundo separado de los adultos, y en la cultura del colegio crean sus propias valores, reglas y jerarquías. El estudio de Coleman (1961), The adolescent society, en highschools estadounidenses, mostraba que lo más importante era lograr popularidad. Este tipo de estudios de mediados del siglo pasado se vuelve pertinente para el caso de nuestros países latinoamericanos en la época actual cuando la mayoría de los jóvenes pasa gran parte de su vida en las escuelas.

En los estudios actuales sobre jóvenes $\mathrm{y}$ tribus urbanas ha tenido gran impacto el concepto de socialidad de Maffesoli (2004), quien postula que nuestra sociedad no es enteramente moderna, racional y civilizada; al contrario, en la sociedad de masas emergen los microgrupos o tribus con su emotividad comunitaria. Maffesoli plantea que en lugar de enfocar la socialización, se debería enfatizar la socialidad. Su concepto de socialidad se basa en el concepto de sociabilidad de Simmel (lo refiere sólo de pasada) quien había postulado en 1917 que "las asociaciones [modernas] están acompañadas de un sentimiento y una satisfacción en el puro hecho de que uno se asocia con otros, y de que la soledad del individuo se resuelve dentro de la unidad: la unión con otros" (SIMMEL, 2002, p. 95). Esta sociabilidad - "el gusto por estar juntos"- es caracterizada también por el mismo Simmel como la "forma lúdica de la asociación" (p. 197). Maffesoli agrega, desde la lectura de Nietzsche (autor a quien refiere extensamente), la noción de los excesos festivos dionisíacos y del predominio de la estética sobre la ética. En nuestros estudios sobre la vida juvenil en las 
escuelas podemos observar la importancia de la sociabilidad y socialidad como veremos.

Sin embargo, sostenemos que la vida juvenil en la escuela, más allá de constituir un espacio de socialización intrageneracional y de sociabilidad, es un espacio de interacción con otros y de subjetivación. El trabajo que nos alertó con relación al concepto de subjetivación fue el libro En la escuela, de Dubet y Martucelli (1998). Una subjetivación, no entendida (sólo) en el sentido actualmente hegemónico, difundido por los seguidores de Foucault, como sujeción del sujeto mediante discursos dominantes, sino en su sentido original en la tradición marxista de Lukács, como emancipación colectiva e individual, la reconquista del sujeto convertido en objeto por el capitalismo (MARTUCCELLI, 2007). Los estudiantes-jóvenes siguen discursos dominantes de las familias, escuelas y medios de comunicación, pero también se emancipan de valores y normas hegemónicos y desarrollan normas, gustos e intereses propios al reflexionar y conversar con sus pares, como trataremos de mostrar.

La perspectiva dominante en los estudios sobre jóvenes -como critican acertadamente Obiols y D'Segni (2006)- los presenta como agentes creadores de nuevas culturas y desestima la investigación psicológica tradicional sobre la adolescencia como una fase de crecimiento, de desarrollo hacia la adultez, con incertidumbres y crisis (ERIKSON, 1974). En nuestras investigaciones los jóvenes hablan de haber madurado a partir de experiencias negativas. Nos parece importante recuperar la perspectiva dinámica y las personas en sus múltiples dimensiones.

El título del presente trabajo más allá de la socialización y de la sociabilidad alude también a un segundo eje de discusión relacionado con nuestras investigaciones sobre el sentido del bachillerato para los jóvenes y sus familias. Múltiples publicaciones enfocan la socialización como reproducción y sostienen que las escuelas reproducen la dominación mediante los mecanismos de selección y de socialización y que los jóvenes de clases populares resisten a la escuela. No queremos ni podemos negar estas afirmaciones ya que nuestras investigaciones se han centrado en estudiantes que asisten a las escuelas y sabemos de los excluidos sólo mediante las voces de los que se quedan en o regresan a la escuela. La integración de estudiantes de sectores urbano-populares y rurales al bachillerato es relativamente reciente en México y enviar a los hijos e hijas al bachillerato con la aspiración de que consigan un mejor trabajo remunerado y después tal vez puedan cursar una carrera profesional a nivel superior constituye una estrategia de reproducción familiar en el contexto de las crisis del empleo. Muchas de las expectativas de ascenso social y económico mediante la escolaridad no se cumplirán. Pero habrá que considerar la persistencia de las expectativas positivas fincadas en la escolaridad por una parte considerable de los sectores populares que aparecen en nuestros estudios.

\section{Los estudios de la línea de investigación}

He desarrollado la línea de investigación sobre jóvenes y escuela principalmente a partir de las tesis de maestría y de doctorado que he dirigido. Nuestra línea comenzó con la tesis de maestría de Irene Guerra y Elsa Guerrero que compara el sentido del bachillerato $^{1}$ para estudiantes del Colegio de Ciencias y Humanidades (CCH), un bachillerato general sostenido por la Universidad Nacional Autónoma de México (UNAM), con el sentido que tiene para estudiantes del Centro de Bachillerato Tecnológico Industrial y de Servicios (CBTIS) en ciudad Nezahualcóyotl, en la zona metropolitana de la ciudad de México.

1- El sistema educativo mexicano se estructura por los siguientes niveles: el preescolar (3 años), la primaria (6 años), la secundaria (3 años) y la educación media superior (3 años, edad 15-17). Dentro de la educación media superior se distingue entre los bachilleratos que certifican la accesibilidad a la educación superior (tradicionalmente llamado preparatorias) y la educación profesional-técnica, aunque ésta ahora también otorga el certificado de bachillerato válido para acceder a la educación superior. Entre los bachilleratos hay bachilleratos generales y bachilleratos tecnológicos. Por su sostenimiento se distinguen entre bachilleratos federales, estatales y universitarios, a cargo directamente de las universidades. 
(GUERRA; GUERRERO, 2004). Personalmente abordé el tema en ocasión de una evaluación curricular de una modalidad de bachilleratos comunitarios indígenas en tres municipios (WEISS, 2006) y Guillermo Tapia (2015) recién lo profundizó al estudiar en su tesis doctoral el sentido de la escolaridad en comunidades ruralurbanas, en particular para estudiantes mujeres, en el Bajío, estado de Guanajuato.

En la investigación de Guerra y Guerrero (2004) sobre los significados de la escuela surgió la importancia del encuentro con compañeros, amigos y novios. El tema de la escuela como espacio juvenil fue profundizado en varias tesis. Job Ávalos (2007) observó en el CCH Sur la vida juvenil en el salón de clase y participó en las conversaciones de un grupo de jóvenes. Beatriz Vega (2013) analizó las conversaciones de estudiantes en el transporte público en el oriente de la ciudad de México.

En su tesis doctoral, Elsa Guerrero (2008) profundizó sobre la experiencia escolar en el $\mathrm{CCH}$, desde la doble perspectiva de ser joven y estudiante. Joaquín Hernández (2007) abordó la formación de la identidad en el bachillerato a partir de esta doble condición y profundizó en los significados del encuentro con amigos y novios y de la sexualidad. Olga Grijalva (2010) investigó las identificaciones que desarrollan los jóvenes de un bachillerato público sostenido por la Universidad Autónoma de Sinaloa, en el puerto de Mazatlán, en sus grupos de pares a través de la ropa, la música y las actividades de estudio y diversión.

El tema de las trayectorias escolares y sus virajes, está presente en las tesis antes mencionadas de Guerrero y de Hernández y constituye el centro de la tesis de doctorado de Irene Guerra (2008), quien estudió los recorridos escolares y laborales de jóvenes de sectores populares, alumnos y egresados del Centro de Bachillerato Tecnológico Industrial y de Servicios en la zona metropolitana del Distrito Federal.

Como se pude apreciar, las investigaciones se realizaron -aprovechando la procedencia de los tesistas en diferentes tipos de escuelas de bachillerato en varias partes del país. He buscado dar cuenta del desarrollo de la línea en conjunto en tres publicaciones: una primera formulación de la línea de investigación (WEISS et al, 2009), en un libro (WEISS, 2012a) que contiene capítulos de cada una de las tesis y en un artículo (WEISS, 2012b) que aborda más bien discusiones teóricas.

\section{Una línea de investigación \\ cualitativa: etnografía, entrevistas, narrativa y hermenéutica}

Para recuperar la voz de los estudiantes, nuestra línea de investigación usa diversos métodos y técnicas cualitativas. La técnica más frecuentemente usada es la entrevista cualitativa, que toma la forma de entrevistas autobiográficas narrativas en los trabajos de Guerrero (2008), Guerra (2008) y Tapia (2015). Algunos trabajos (GUERRER0, 2008; TAPIA, 2015) usan adicionalmente cuestionarios o una cédula al final de la entrevista.

Otros trabajos usan la observación etnográfica y la conversación con los sujetos en sus ambientes naturales, en el patio y los jardines escolares (HERNÁNDEZ, 2008; GRIJALVA, 2010; TAPIA, 2015) o la observación participante como Ávalos (2007) quien convivió con un grupo de estudiantes del CCH Sur durante un semestre en sus clases de lectura y redacción y de biología y participó en sus conversaciones dentro y fuera del salón de clases. Vega (2013) presenció las conversaciones en voz alta de estudiantes en el transporte público en sus viajes de la casa a la escuela y de regreso. Para la evaluación curricular de Weiss (2006) visitamos tres escuelas, de lengua zapoteca, mazateca y mixteca respectivamente, por una semana cada una, con un grupo de investigadores.

Nuestra línea de investigación cualitativa se inscribe en buena parte en la tradición etnográfica del Departamento de Investigaciones Educativas (DIE) del Centro de Investigación y de Estudios Avanzados del Instituto Politécnico 
Nacional (Cinvestav) que propuso "documentar lo no documentado", es decir, la vida escolar (ROCKWELL, 1987, 2009, p. 21) no documentada por los informes burocráticos, pero tampoco por la investigaciones pedagógicas, sociológicas, psicológicas o antropológicas que se acercan con un marco teórico preconcebido a partir del cual constatan la presencia o ausencia de determinados fenómenos. El enfoque etnográfico del DIE-Cinvestav nació desde la perspectiva de vida cotidiana en términos de Schütz, Gramsci, Heller y Berger y Luckmann (ROCKWELL, 1987), así como desde la perspectiva del interaccionismo simbólico (PARADISE, 1994). Actualmente enfatiza la apropiación de recursos culturales, una apropiación que integra simultáneamente la cultura -concebida como compleja, múltiple, situada e histórica- como algo que constriñe y a la vez posibilita, enfatiza la agencia activa y transformadora de los sujetos y la multiplicidad de flujos culturales (ROCKWELL, 2001). A diferencia de la etnografía estadounidense centrada en minorías, busca fijar su atención en la vida escolar de los sectores populares en un sentido amplio, sectores que en América Latina constituyen la mayoría de la población (ROCKWELL, 1998, 2001).

Como ha señalado en diversas ocasiones Rockwell (1987, 2009), en el enfoque cualitativo el análisis no es una fase posterior al levantamiento de datos sino está presente desde la formulación de las preguntas de investigación y a lo largo del trabajo de campo como continua modificación y cambio de las preguntas iniciales. No obstante, el trabajo más difícil llega cuando se trata de analizar el cúmulo de registros generados. Considero que el enfoque de esta fase del trabajo es fundamentalmente hermenéutico: se busca comprender las transcripciones como si fueran un texto de varias voces. Se trata de comprender determinado significado en el contexto de otros significados simultáneos (como partes de un todo; el círculo hermenéutico como configuración de sentidos), y se trata de modificar nuestras anticipaciones de sentido hasta que logren ser interpretaciones pertinentes a lo que dice el texto (la espiral hermenéutica) (WEISS, 2005).

No puedo comprender nada si no parto de ciertas anticipaciones de sentido -pre-juicios o juicios previos, como los llama Gadamer (2005)-, anticipaciones que contienen elementos de nuestra experiencia de vida, pero también elementos teóricos. No se trata de imponer nuestra experiencia y forma de ver, sino de escuchar y comprender lo que el otro, el texto, tiene que decir; se trata de comprender en primera instancia la otredad. Luego, en varias vueltas de lectura y de anticipaciones modificadas, logro interpretaciones (juicios) más pertinentes.

Actualmente está otra vez de moda señalar que el trabajo en cuestión se basa en la teoría fundamentada de Glaser y Strauss (1967). Estos autores promovieron, en contra del enfoque hipotético deductivo que prevalecía en su época, una concepción inductiva de generación de categorías teóricas -si bien a lo largo del texto del libro reconocen el interjuego constante entre observables y teorías preexistentes. A mi ver partimos de algunas teorizaciones iniciales que anticipamos como pertinentes; en el transcurso del análisis podemos en algunos casos precisarlas, en otros tenemos que encontrar teorizaciones más pertinentes. Muchas tesis de doctorado pretenden generar nuevas categorías teóricas en una sola investigación, pero los ejemplos de Glaser y Strauss mismos, indican que eso se logra a través de líneas de investigación sostenidas. Es así como pudimos en nuestra línea generar y dar vida a los conceptos de la escuela como espacio juvenil y de subjetivación.

Considero exagerado -promovido por programas de análisis de datos cualitativosel énfasis actual en las categorías analíticas o intermedias entre grandes teorías y los observables concretos. La herramienta tradicional de la hermenéutica de textos y de la etnografía es más bien la descripción, una descripción que es a la vez condensación y paráfrasis, generación de nuevos significados 
como dice Barthes (1966), o traducción como dicen Velasco y Díaz (2007). En las investigaciones particulares, dialogamos con diversas teorías a partir de nuestros datos empíricos, luego buscamos exponer lo encontrado en una argumentación coherente, sustentada teórica y empíricamente, y buscamos que lo expuesto sea considerado por otros como una aportación al conocimiento, y a veces como nueva propuesta de teorización.

\section{El sentido de la escolaridad}

En México la matrícula de la educación media superior ha crecido de manera continua de 2,1 millones de alumnos en 1990-1991 a 4,4 millones en 2012-2013 -muy por encima del aumento poblacional de los últimos veinte años- basado en el crecimiento de la educación secundaria (media básica), en el aumento de la oferta institucional y sobre todo de la demanda de las familias y sus hijos. El crecimiento es apoyado por una política de otorgamiento de becas, que actualmente cubre a 50\% de los matriculados en la educación pública de ese nivel con montos de 650 y 1055 pesos mensuales; hoy en día casi todos los egresados de la educación secundaria mexicana (educación media básica) se inscriben al primer año de la educación media superior. (WEISS, 2014) Aun así, la población escolarizada de 17 años llega sólo a 57.9\% en 2010, muy por debajo de la de Argentina y Brasil donde asciende a $82.5 \%$ y 75.9\% respectivamente (ITZCOVICH, 2014). Un 40\% de los que se inscriben al primer grado de primaria abandona la escuela antes de terminar la secundaria y de los que se inscriben en primer grado de la educación media superior un 40\% la abandona antes de concluirla, de manera que de los 100 que iniciaron la primaria en 1999 sólo 36 lograron terminar la media superior en 2010 (ENDEMS, 2012).

Es impactante el cambio en el acceso a la escolaridad en zonas rurales e indígenas, sobre todo con relación a las mujeres. En 1993 realizamos con mi colega Justa Ezpeleta un estudio sobre la escuela rural multigrado en los estados de Guerrero y Oaxaca (EZPELETA; WEISS, 2000). Muchas niñas no terminaban su estudios de primaria, aun cuando habían llegado sólo a $4^{\circ}$ grado, por haber comenzado tarde sus estudios de primaria al haber cuidado a sus hermanos pequeños o por haber reprobado algún grado; a la edad de 13 años eran sacadas de la escuela por sus padres al estar en edad reproductiva y expuestas a los peligros del rapto por varones en el camino.

En cambio en 2006, en el estudio del bachillerato en tres comunidades indígenas de Oaxaca, el número de mujeres era igual que el de hombres, en una escuela incluso era mayor. En zonas de pobreza las familias de los y las estudiantes reciben becas; desde 2002 se otorgan también para los estudios de bachillerato y con un monto mayor para las mujeres. La asistencia a la escuela es considerada equivalente a un trabajo remunerado y forma parte del ingreso familiar; como me dijo un padre: "Nosotros trabajamos en el campo, ellos en la escuela" (WEISS, 2006, p. 90). Hubo un cambio profundo en los patrones culturales.

La investigación de Guerra y Guerrero (2004) sobre el significado del bachillerato, muestra que en un bachillerato afiliado a la universidad el sentido predominante que otorgan los estudiantes es el ingreso a las carreras de educación superior; en un bachillerato tecnológico de la zona conurbada del Distrito Federal, también tiene este sentido para la gran mayoría, si bien estos estudiantes no están seguros de obtener el ingreso a las instituciones superiores, ya sea por su situación económica o porque sus calificaciones $\mathrm{y}$ habilidades académicas no lo permitan. Incluso en el bachillerato intercultural comunitario, en municipios rurales indígenas de Oaxaca, alrededor de un tercio de los estudiantes piensa seguir estudiando -las carreras que más anhelan son: médico, maestro, abogado, enfermera, ingenierías, contabilidad e informática-, otro tercio no está tan seguro si puede seguir y todos piensan trabajar mientras estudian si 
no obtienen una beca. A las mujeres que no piensan estudiar, el certificado les sirve para obtener un mejor trabajo, por ejemplo, en una tienda en la ciudad en lugar de ser sirvientas en casas, y a los hombres para conseguir un empleo urbano en Estados Unidos, en lugar de los tradicionales trabajos agrícolas temporales o de albañil. (WEISS, 2006).

Para los jóvenes de zonas populares, el certificado del bachillerato también abre la posibilidad de empleos formales - "ahorita para cualquier trabajo más o menos están pidiendo, por lo menos, el bachillerato" (GUERRA, 2009, p. 188) - que antes se conseguían con el certificado de secundaria. De los egresados entrevistados por Guerra (2008) apenas la mitad había logrado un empleo formal, lo lograron después del bachillerato y en algunos casos sólo después de cursar los estudios superiores.

En los sectores populares, urbanos, ruralurbanos e indígenas uno de los principales motivos para estudiar de las familias y sus hijos es superar la condición social y económica: tener una vida mejor. Esta mejora económica a veces no sólo se plantea en el plano personal, sino como parte de un esfuerzo familiar. Son frecuentes los casos donde los jóvenes pudieron estudiar gracias a un hermano mayor que tuvo que dedicarse a trabajar para poder ayudar a los ingresos familiares o en los que ellos mismos proyectan, en el futuro, sostener el estudio de sus hermanos menores con un mejor trabajo; se plantean retribuir los esfuerzos de la familia. (GUERRA, 2009; TAPIA, 2015). En el contexto de la transición rural- urbana en el Bajío, las familias incorporan la escolaridad de los hijos entre sus estrategias de reproducción ${ }^{2}$ (TAPIA, 2015). Aún con las becas sigue habiendo familias para las que el costo del bachillerato es demasiado oneroso, pero la gran mayoría de familias con hijos que lograron terminar la secundaria está dispuesta a afrontar el gasto. Los problemas surgen cuando salen imprevistos:

2- Concepto usado por Cragnolino (2002) en el contexto de la zona rural al norte de la ciudad de Córdoba, Argentina. una enfermedad grave de algún miembro o el padre que abandona la familia (GUERRA, 2009).

Los motivos para cursar el bachillerato no sólo son económicos, también se menciona en sectores urbanos populares y rurales el prestigio social: ser alguien en la vida ${ }^{3}$ sobre todo si se logra ser profesionista. Reiteradamente aparecen motivos de logro personal, es decir, demostrar a sí mismos y a la familia que sí pude.

Entre mujeres de sectores populares y con mayor fuerza en zonas rurales, está presente la motivación de "superar la condición de género" como la llamaron Guerra y Guerrero (2004), por unas parte como reacción a la discriminación por parte de familiares y vecinos y por otra parte en un sentido propositivo: serán futuras madres más instruidas. Tapia (2015) muestra que en el Bajío - en una zona de transición rural - urbana y de un catolicismo arraigadoaún esta difundido entre padres y abuelas el modelo cultural ${ }^{4}$ : para qué estudias si de todos modos te vas a casar. Sin embargo, las madres apoyan a sus hijas para que asistan a la escuela para poder valerse por sí mismas, en caso de que el marido no cumpla con las obligaciones. Estas jóvenes desplazan el noviazgo, el matrimonio y la procreación de hijos - usual en la edad de 16 a 19 años- hasta terminar cuando menos el bachillerato e incluso planean primero tengo $\mathrm{mi}$ carrera. Es decir, el bachillerato, al romper con el encierre doméstico y abrir las posibilidades de un trabajo remunerado, contribuye a la emancipación femenina.

Evidentemente estas motivaciones positivas no aparecen con igual fuerza en todos los alumnos. Hay estudiantes que asisten porque sus familias los envían y van a la escuela para encontrarse con sus compañeros. Guerra (2008), al estudiar los recorridos escolares y laborales de los estudiantes del bachillerato tecnológico, distingue entre los que se adhirieron a la cultura escolar y los

3- La frase "ser alguien en la vida" se escucha en varios países de América Latina entre los jóvenes, padres y madres de familia de sectores populares.

4- La noción de modelo cultural proviene de Quinn y Holland (1987). 
que nunca desarrollaron una adhesión a los valores y normas de la escuela y consideraron más importante el trabajo y, en el caso de las mujeres, la vida en pareja o los hijos ${ }^{5}$, y los que entraron en conflicto con los valores y normas de la escuela y prefirieron la vida juvenil en la calle. En los casos de abandono escolar las razones o motivos se entrelazan: el joven tiene dificultades académicas; en su barrio o en la escuela se une a un grupo que se dedica poco o nada a los estudios y, en consecuencia, falta a clases, no atiende sus deberes y reprueba crecientemente materias; en este contexto cualquier incidente, una riña, una enfermedad, una oferta de trabajo temporal atractiva, otra reprobación o una mala palabra de un maestro impulsan al joven a abandonar la escuela o a ser expulsado (GUERRA, 2009).

\section{La escuela como espacio de vida juvenil}

Nos causó gran impacto encontrar en las entrevistas para el estudio sobre el sentido del bachillerato de Guerra y Guerrero (2004) con frecuencia expresiones como las siguientes: estoy en la escuela para ver a mis amigos, encontrarme con mi novia, en casa me aburro. Para dar cuenta de estas expresiones creamos la categoría "la escuela como espacio de vida juvenil". Este hallazgo fue tan sorpresivo para nuestra mirada pedagógica y sociológica que decidimos profundizar en el tema de la vida juvenil y su importancia en la experiencia escolar y en la construcción de la identidad en las tesis siguientes.

En el bachillerato afiliado a la universidad nacional en la ciudad de México, la vida juvenil se despliega en toda su amplitud. En la explanada y los jardines se pueden encontrar jóvenes estudiosos comparando sus

5- En la Encuesta Nacional de Deserción en la Educación Media Superior las dos razones de abandono que siguen en importancia a la falta de dinero fueron en el caso de las mujeres: "se embarazó o tuvo un hijo" (23.8\%) y "se casó" (22.9\%) (ENDEMS, 2012, p. 65). notas, jóvenes con estética dark intercambiando música y otros con peinados y vestidos al estilo rastafari tocando sus bongos; se observan claras manifestaciones eróticas y algunos estudiantes consumen a escondidas alcohol (HERNÁNDEZ, 2008). La vida juvenil no se limita a los espacios intersticiales de la actividad académica (es decir, antes, entre y después de clases), sino que se despliega también durante el tiempo de clase, sustrayendo tiempo a la actividad escolar para bromas o intercambios (de tonos y juegos en celulares, música y cómics, entre otros) hasta las diversas conversaciones que surgen espontáneamente sobre los temas más variados, especialmente durante los trabajos grupales, como muestra el estudio de Ávalos (2007).

La doble condición de ser estudiante y ser joven es central en nuestros estudios. Las diversas posibilidades de vida juvenil suelen competir con los requerimientos escolares. Grijalva (2010) encuentra en el patio de un bachillerato dependiente de la universidad en el puerto de Mazatlán, grupos de alumnos que privilegian el trabajo escolar (los menos), los que privilegian la diversión y la vida juvenil (los más) y grupos que combinan estratégicamente la diversión con el trabajo escolar. Vega (2013) señala que en las conversaciones que sostienen los estudiantes con compañeros y amigos en el transporte público hacia y desde la escuela, las tareas y las calificaciones tienen un lugar preponderante. Además, sorprende la agresividad con la que negocian sus calificaciones con los profesores según relatan a sus compañeros. Dubet y Martuccelli (1998) sostienen que entre estudiantes franceses del bachillerato la estrategia es la lógica de interacción predominante para decidir qué cursos tomar y cuáles materias privilegiar. Los estudiantes mexicanos estrategas invierten el tiempo necesario para conseguir sus fines: sean éstos lograr buenas calificaciones para poder entrar a la universidad y a la carrera de su elección, sean éstos obtener el mínimo necesario para pasar los exámenes extraordinarios. 
Sociabilidad, socialización, subjetivación

En varios de nuestros estudios (HERNÁNDEZ, 2008; ÁVALOS, 2007; GRIJALVA, 2010) podemos observar la importancia de la sociabilidad (SIMMEL, 2002), del disfrute de estar juntos con compañeros, amigos y novios. También se manifiesta la socialidad (MAFFESOLI, 2004), que está presente en el relajo, los juegos físicos y las bromas. Las fiestas y los eventos musicales son de lo más importante para muchos jóvenes; en la escuela se comentan las experiencias en los últimos eventos y fiestas, además, se planea la asistencia a los próximos. Sin embargo, las experiencias de los jóvenes no se limitan al hedonismo ni a la sociabilidad. En tanto estudiantes tienen obligaciones escolares y aún la convivencia juvenil y los encuentros con compañeros, amigos y parejas significan más que sólo vibrar juntos.

Las investigaciones de nuestra línea abordan escasamente el tema de la socialización intergeneracional de los jóvenes estudiantes por parte de las familias y de la institución escolar. En cambio podemos observar cómo diferentes grupos de estudiantes de bachillerato desarrollan sus propias normas y valores respecto al comportamiento con los maestros y a la dedicación a las tareas, al look, a la música aceptable y al tipo de diversiones preferidas (GRIJALVA, 2010; ÁVALOS, 2007). En este sentido podemos hablar con Coleman (1961) de una socialización intrageneracional en oposición a la intergeneracional pretendida de los adultos. Desde una perspectiva crítica podemos constatar que en cuestiones de look los estudiantes se identifican con determinadas culturas juveniles y sus dictados de moda, en términos de Foucault se constituyen como sujetos en tanto son sujetados por los discursos de las industrias de consumo juveniles. Pero también es interesante observar el desarrollo de un gusto propio entre las mujeres estudiantes de más edad en el bachillerato en el puerto de Mazatlán: ellas destacan la forma personal en la que combinan ciertos atuendos y accesorios, considerando en sus decisiones las características de su cuerpo y personalidad. (GRIJALVA, 2010).

El concepto de socialización, al enfatizar la transmisión de valores y normas de la sociedad a sus miembros y la integración del actor a los valores y normas de determinado grupo o institución, no es capaz de dar cuenta de la riqueza de las vivencias de interacción con otros y de la reflexión sobre ellas. Más allá del alcance de los conceptos de sociabilidad y de socialización se requiere de una conceptualización que permita abordar la individuación de la persona. Propongo rescatar el concepto de subjetivación con las siguientes características (WEISS, 2012 b):

Las normas y valores no se absorben, se modifican en los procesos de interiorización y apropiación (CHARTIER, 1991 apud ROCKWELL, 2005); se desarrolla la capacidad de reflexionar (GIDDENS, 1997) desde el self sobre las distintas demandas y sobre la posición propia en ellas (MEAD, 1934 apud HERNÁNDEZ, 2008); de ahí nacen la emancipación de las normas y valores dominantes y el desarrollo de normas y valores propios (MARTUCCELLI, 2007), así como el desarrollo de gustos, intereses y capacidades propios (TAYLOR, 1996) que fundamentan la agencia del sujeto, la capacidad humana de hacer (BRUNER, 1990) y decidir (KIERKEGAARD apud HONG, H.; HONG, E., 2000).

Estos procesos de subjetivación se desarrollan a través de diversas prácticas mediante la interacción con otros -sobre todo con compañeros, amigos, y novios- $\mathrm{y}$ mediante la conversación sobre sus vivencias, pero también se vuelven observables cuando hablan de sus reflexiones sobre sus recorridos y señalan que han madurado, como veremos en los apartados siguientes.

\section{Las experiencias y las conversaciones con otros}

A diferencia de los estudios sobre culturas juveniles, donde destaca el "otro antagonista" -la 
"alteridad radical" que a su vez permite construir un sentimiento de nosotros (REGUILLO, 2000, p. 41)-, en nuestros estudios prevalece el otro semejante. El otro más importante es la pareja amorosa o sexual. Los estudiantes en el CCH en la ciudad de México establecen diferentes tipos de relaciones: como chocolate (tomándose de las manos), amigovio (medio amigo, medio novio), free (encuentro erótico o sexual casual) o como novio (HERNÁNDEZ, 2008). Los Bachilleratos Interculturales Comunitarios constituyen un espacio público inédito para el encuentro entre jóvenes mujeres y hombres en los municipios indígenas. Las relaciones eróticas son más reservadas pero observables. (HERNÁNDEZ; WEISS, 2010). El conocimiento práctico del otro pasa por la sensualidad corporal, pero también por compartir, abrirte al otro, por el apoyo recíproco, el ensayo y el error. En las relaciones amorosas los jóvenes aprenden a manejar los vínculos sociales de intimidad, confianza, cuidado del otro e incertidumbre amorosa, así como las capacidades de expresar los afectos, descubrir otras maneras de sentir y atender las necesidades del otro, como muestra Hernández (2008).

Hernández (2008) enfatiza el ensayo y el error, la exploración de prácticas, comportamientos, sentimientos y pensamientos. El concepto de experiencia implica la noción de experimentación, como se puede apreciar en los escritos del ámbito anglosajón, donde la noción de experiencia alude desde Bacon a lo empírico sensual corporal y a la prueba de experimentación y alcanza con Dewey ser concebida como reflexión sobre la acción. Dayrell en su estudio sobre jóvenes y escuela en Brasil enfatiza que sus aventuras $y$ experimentaciones implican retos personales: las múltiples experimentaciones de los jóvenes "buscan superar la monotonía de lo cotidiano y procuran aventuras y excitaciones” (DAYRELL, 2007, p. 1117).

Pero los jóvenes no solo viven aventuras, también reflexionan, aprenden de sus experiencias, les sirven para conocerse mejor a sí mismos y trazar caminos y proyectos. El término alemán para experiencia es Erfahrung y contiene la noción existencial de diversas vivencias en los caminos de la vida: fahren significa viajar. Erfahrung suele referirse a los eventos memorables durante la vida y a veces el concepto de experiencia se confunde con la transferencia de conocimientos de generaciones anteriores a los jóvenes. Pero, como señala Gadamer (2005) con respecto al concepto de Erlebnis (vivencia): las vivencias las tiene que realizar cada uno, no solo en el sentido positivo sino también en el negativo, las vivencias dolorosas y las equivocaciones; las vivencias son, sobre todo, vivencias del Tú y de comprensión del Tú, es decir, de la convivencia con otros humanos. Las vivencias (y yo diría las experiencias) son, por un lado, inseparables del mundo en que vivimos y de sus tradiciones culturales, a la vez que requieren de la conciencia de la existencia como persona en la finitud de la vida. Parafraseando a Gadamer podríamos decir: para que las vivencias (individuales y colectivas) se conviertan en experiencias, es necesario tomar conciencia del mundo cultural e histórico en el que vivimos y de la historicidad y vulnerabilidad de nuestra vida como persona. El concepto de experiencia implica la reflexión sobre las vivencias. Y una de las formas de reflexión privilegiada es la conversación.

Bourdieu ya caracterizaba a los estudiantes herederos como conversadores. Dubet y Martuccelli confirman: "Así, en el liceo, los alumnos consagran lo esencial de su tiempo libre... a practicar el arte de la conversación" (DUBET; MARTUCCELLI, 1998, p. 334). En México los jóvenes no suelen juntarse en cafés pero en las escuelas sí. Ávalos (2007) relata algunas de las conversaciones dentro y fuera del salón de clase: los jóvenes hablan de sus vivencias en la última fiesta y de la próxima a organizar, discuten sobre los grupos musicales o eventos deportivos, comentan algunos programas de televisión y hablan de sus inquietudes y experiencias afectivas o sexuales. Vega (2013) identifica que en las conversaciones entre jóvenes bachilleres en el transporte público predominan temas del ámbito juvenil como los 
amigos y compañeros, las relaciones con el otro género y noviazgos, y la música y las fiestas; y obviamente - realizándose en el transporte hacia y desde el escuela - se tratan temas escolares como las tareas, entrar o no entrar a clases, las calificaciones y las relaciones con los profesores. En menor medida se tematiza la relación con la familia en términos de apoyos y conflictos. Escasas son las menciones de temas relacionados con el trabajo y la religión. Es notable -al igual que en las conversaciones atestiguadas por Ávalos (2007) - la ausencia de temas políticos.

Las conversaciones entre los estudiantes, aun cuando versan sobre temas triviales, les permiten conocer otras opiniones y son una forma de reflexión, como señala Hernández (2008). Al platicar en confianza entre ellos, los jóvenes pueden mostrarse de manera más intima y conocer puntos de vista diferentes al suyo. A las amigas "sí les puedo contar todo mi sentir y todo.... y si estás haciendo algo donde la estés regando pues también te lo van a decir para que te des cuenta" dice una joven entrevistada (WEISS et al, 2009, p. 90). Las conversaciones de los jóvenes con sus pares dan cuenta de las vivencias que tienen en otros ámbitos, por ejemplo el familiar; así la conversación se convierte en un vehículo para la interconexión (DREIER, 2010) de distintos ámbitos y prácticas de los jóvenes.

\section{Recorridos y retornos}

En el bachillerato, tanto las escuelas como las familias otorgan a los jóvenes un mayor margen de libertad y decisión propia, si bien eso no es homogéneo entre las diferentes familias ni entre los diferentes tipos de escuela. La mayor capacidad de decisión propia de estos estudiantes permite vivir la vida juvenil en sus diferentes manifestaciones y experimentarla con sus riesgos, y los riesgos siempre han atraído a los jóvenes (WEISS et al, 2009). Hay muchas libertades: "muchísimas, por ejemplo, aquí tú ves a la gente fumando... por ejemplo si quieres entrar a tus clases, si no quieres no entras, porque aquí no hay prefectos..."
(GUERRERO, 2012, p. 132). Después de un ingreso lleno de expectativas positivas suele surgir el atractivo del espacio juvenil: "el ambiente, ¿no?, te va jalando, te gusta pues, andar conviviendo y todo" (p. 132). El grupo de amigos invita a faltar a clases (GUERRERO, 2008; HERNÁNDEZ, 2008).

También poreso muchosjóvenes terminan con malas calificaciones y otros abandonan la escuela. Pero no pocos logran el retorno: estudiantes del CCH se dedican otra vez más a los estudios en los últimos semestre para poder concluir el bachillerato (GUERRERO, 2008) y jóvenes del bachillerato tecnológico retornan a la escuela después de haberla abandonado y de pasar un tiempo en la banda y el alcohol o en experiencias poco gratas en trabajos informales (GUERRA, 2009). En los virajes en la dirección del curso de vida -usando un concepto acuñado por Elder (1994) y referido por Guerrero (2008)el aprendizaje más significativo para algunos estudiantes parece haber sido el siguiente: "todas estas experiencias me sirvieron para hacerme más responsable... me sirvió mucho porque, este, híjole! Fue una época en la que me hice responsable" (GUERRERO, 2012, p. 148).

Es significativo el uso de palabras como adquirir responsabilidad, reflexionar y madurar por parte de los estudiantes (HERNÁNDEZ, 2008; GUERRERO, 2008). Dubet y Martuccelli (1998) señalan que para crecer, para pasar de niño a adolescente (y a adulto, agregaríamos), un factor importante es la madurez y las características emocionales de seguridad e independencia asociadas a ella. Hernández (2008) considera -usando la expresión de Beck y Beck-Gernsheim (2003)- que hacerse cargo de sí mismo y de su vida es una parte importante del proceso de individuación.

\section{A modo de cierre}

Habría mucho más que contar sobre la línea de investigación pero el espacio disponible en una revista es limitado.

Enfocamos a los sujetos de nuestra investigación en su triple condición de estudiantes, 
de jóvenes-adolescentes y de hijos/hijas de familia. Los estudiantes que aparecen en los trabajos de nuestra línea de investigación pasan gran parte de su tiempo escolar en las interacciones con sus pares, les gusta estar asociados con sus compañeros y amigos (sociabilidad) y divertirse con ellos (socialidad). En cada grupo establecen sus propias normas y valores (socialización entre pares). A la vez, ser estudiante requiere seguir ciertas normas de la escuela y de la sociedad de adultos (socialización inter-generacional).

Tanto las familias como los establecimientos escolares suelen otorgar a los estudiantes del nivel medio superior mayor libertad para decidir sobre el uso de su tiempo. Esta mayor libertad es consecuencia y requisito de un creciente proceso de subjetivación de los jóvenes, del desarrollo de gustos, intereses y capacidades, de la emancipación de normas y valores, de la creciente reflexión sobre las exigencias y necesidades de otros, y de la creciente capacidad de elección y decisión.

La subjetivación no es -en la mayoría de los casos- un proceso solitario. Los jóvenes comparten con compañeros, amigos y novios vivencias y conversan sobre ellas. Estas conversaciones se realizan por el placer de conversar (sociabilidad), pero son también una forma de reflexión que alienta la subjetivación. Al reflexionar sobre las vivencias, éstas se constituyen en experiencias. Conocer y comprender a otros les permite conocerse y comprenderse mejor a sí mismos e ir construyendo sus identificaciones. A través de múltiples vivencias y experiencias, los jóvenes se socializan, en el sentido de saber manejar las normas y valores en diferentes ámbitos de la vida, y se subjetivan al elaborar valores, normas y decisiones propias; a la vez construyen sus identidades en diferentes ámbitos de su vida y esbozan sus proyectos (siempre temporales).

Los jóvenes exploran diferentes formas de vivir y cometen errores, pero también reflexionan y cambian. Por ello creemos importante recuperar el concepto del adolescente que crece, no como una etapa en una concepción evolutiva, sino como proceso continuo. No nos interesa el proceso intrasubjetivo, sino el proceso que se gesta en prácticas y vivencias con otros y que se convierte, a través de las conversaciones intersubjetivas y la reflexión, en experiencias intra-subjetivas. Estamos interesados en el sujeto psicológico, social y cultural, es decir, en la persona. Para futuras investigaciones educativas propongo recuperar el concepto de persona - más allá de los conceptos de actor, sujeto o agente - y recuperar el concepto de (auto) formación - más allá de los conceptos de socialización y de subjetivación.

\section{Referencias $^{6}$}

ÁVALOS, Job. La vida juvenil en el bachillerato: una mirada etnográfica. 2007. 172 p. Tesis (Maestría en Ciencias en Investigaciones Educativas) - Departamento de Investigaciones Educativas. Centro de Investigación y de Estudios Avanzados del Instituto Politécnico Nacional - DIE-CINVESTAV, México, DF, 2007.

BARTHES, Roland. Critique et verité. Paris: Seuils, 1966.

BECK, Ulrich; BECK-GERNSHEIM, Elisabeth. La individualización: el individualismo institucionalizado y sus consecuencias sociales y políticas. Madrid: Alianza, 2003.

BRUNER, Jerome. Actos de significado: más allá de la revolución cognitiva. Madrid: Alianza, 1990.

CHARTIER, Roger. The cultural origins of the French Revolution. Durham: Duke University Press, 1991.

COLEMAN, James. The adolescent society. Nueva York: Free Press, 1961.

6- Las publicaciones de partes de las tesis como capítulos en el libro Jóvenes y bachillerato y como artículos en revistas no se reportan aquí por falta de espacio. Artículos derivados de las tesis pueden encontrarse con los nombres de los autores en Redalyc o SciELO. 
CRAGNOLINO, Elisa. Trayectorias educativas en familias de origen campesino del norte cordobés. Cuadernos de Educación, Córboda, n. 2. p. 151-165, dic. 2002.

DARELL, Juarez. A escola 'faz' as juventudes? Reflexões em torno da socialização juvenil. Educação \& Sociedade, Campinas, v. 28, n. 100, p. 1105-1128, out. 2007.

DREIER, Ole. Trayectorias personales de participación a través de contextos de práctica social. In: PÉREZ CAMPOS, Gillberto et al. (Coords.). Psicología cultural. v. 1. México, DF: UNAM-FES Iztacala, 2010. p. 81-128.

DUBET, François; MARTUCCELLI, Danilo. En la escuela: sociología de la experiencia escolar. Buenos Aires: Losada, 1998.

ELDER, Glen. Time, Human agency, and social change: perspectives on the life course. Social Psychology Quarterly, v. 57, n. 1, p. 4-15, 1994.

ENDEMS - Encuesta Nacional de Deserción en la Educación Media Superior. Reporte de la Encuesta Nacional de Deserción en la Educación Media Superior. México, DF: SEP - Consejo para la Evaluación de la Educación del Tipo Medio Superior, 2012.

ERIKSON, Erik. Identidad, juventud y crisis. Buenos Aires: Paidós, 1974.

EZPELETA, Justa; WEISS, Eduardo. Cambiar la escuela rural: evaluación cualitativa del programa para abatir el rezago educativo. México, DF: DIE-CINVESTAV, 2000.

GADAMER, Hans-Georg. Verdad y método: fundamentos de una hermenéutica filosófica. Salamanca: Sígueme, 2005.

GIDDENS, Anthony. Modernidad e identidad del yo: el yo y la identidad en la época contemporánea. Barcelona: Península, 1997.

GLASER, Barney; STRAUSS, Anselm. The discovery of grounded theory: strategies for qualitative research. New York: Aldine de Gruyter, 1967.

GRIJALVA, Olga. Las apariencias como fuente de las identificaciones en la construcción de las identidades y en la conformación de grupos juveniles. 2010. 215 p. Tesis (Doctorado en Ciencias en Investigaciones Educativas) - Departamento de Investigaciones Educativas. Centro de Investigación y de Estudios Avanzados del Instituto Politécnico Nacional - DIE-CINVESTAV, México, DF, 2010.

GUERRA, Irene. Trayectorias escolares y laborales de jóvenes de sectores populares: un abordaje biográfico. 2008. 292 p. Tesis (Doctorado en Ciencias en Investigaciones Educativas) - Departamento de Investigaciones Educativas. Centro de Investigación y de Estudios Avanzados del Instituto Politécnico Nacional - DIE-CINVESTAV, México, DF, 2008.

GUERRA, Irene. Trayectorias formativas y laborales de jóvenes de sectores populares: un abordaje biográfico. México: Anuies, 2009.

GUERRA, Irene; GUERRERO, Elsa. ¿Qué sentido tiene el bachillerato? Una visión desde los jóvenes. México: Universidad Pedagógica Nacional, 2004.

GUERRERO, Elsa. Los estudios de bachillerato: un acercamiento a la perspectiva juvenil. 2008. 306 p. Tesis (Doctorado en Ciencias en Investigaciones Educativas) - Departamento de Investigaciones Educativas. Centro de Investigación y de Estudios Avanzados del Instituto Politécnico Nacional - DIE-CINVESTAV, México, DF, 2008.

GUZMÁN, Carlota; SAUCEDO, Claudia. La investigación sobre alumnos en México: recuento de una década (1992-2002). In: DUCOING, Patricia (Coord.). Sujetos, actores y formación. Tomo II. México, DF: COMIE/IPN/Grupo Ideograma, Colección La investigación educativa en México, 2005. p. 641-832.

HERNÁNDEZ, Joaquín. El trabajo de los estudiantes de bachillerato: reflexividad, voces y marcos morales. México: Universidad Pedagógica Nacional, 2008.

HERNÁNDEZ, Joaquín. La formación de la identidad en el bachillerato: reflexividad y marcos morales. 2007. 162 p. Tesis (Doctorado en Ciencias en Investigaciones Educativas) - Departamento de Investigaciones Educativas. Centro de Investigación y de Estudios Avanzados del Instituto Politécnico Nacional - DIE-CINVESTAV, México, DF, 2007. 
HERNÁNDEZ, Joaquín; WEISS, Eduardo. Amor y sexualidad entre jóvenes de la escuela media en México. Cuadernos de Educación, Córdoba, v. 8, n. 8, p. 245-256, oct. 2010.

HONG, Howard; HONG, Edna. The essential Kierkegaard. Princeton: Princeton University Press, 2000.

ITZCOVICH, Gabriela. La expansión educativa en el nivel medio América Latina, 2000-2010. Cuaderno SITEAL, Buenos Aires, n. 19, jun. 2014. Disponible en: <http://www.siteal.iipe-oei.org/sites/default/files/siteal_cuaderno_19_gi_nivel_medio_v2.pdf> Acceso en: 11 mayo 2015.

MAFFESOLI, Michel. El tiempo de las tribus: el ocaso del individualismo en las sociedades posmodernas. México, DF: Siglo XXI, 2004.

MARTUCCELLI, Danilo. Gramática del individuo. Buenos Aires: Losada, 2007.

MEAD, George. Espíritu, persona y sociedad. Barcelona: Paidós, 1999.

OBIOLS, Guillermo; DI SEGNI, Silvia. Adolescencia, posmodernidad y escuela. Buenos Aires: Novedades Educativas, 2006.

PARADISE, Ruth. Etnografía. ¿Técnicas o perspectiva epistemológica? In: RUEDA, Mario; DELGADO, Gabriela; JACOBO, Zardel. La etnografía en educación: panorama, prácticas y problemas. México, DF: CISE-UNAM, 1994. p. 73-81

QUINN, Naomi; HOLLAND, Dorothy. Culture and cognition. In: QUINN, Naomi; HOLLAND, Dorothy (Eds.) Cultural models in language and thought. New York: Cambridge University Press, 1987. p. 3-40.

REGUILLO, Rossana. Emergencia de culturas juveniles: estrategias de desencanto. Bogotá: Norma, 2000.

ROCKWELL, Elsie. Caminos y rumbos de la investigación etnográfica en América Latina. Cuadernos de Antropología Social, Buenos Aires, n. 13, p. 53-64, 2001.

ROCKWELL, Elsie. Ethnography and the commitment to public schooling: a review of research at the DIE. In: ANDERSON, Gary L.; MONTERO-SIEBURTH, Martha. Educational qualitative research in Latin America: the struggle for a new paradigm. New York; London: Garland, 1998. p. 3-33.

ROCKWELL, Elsie. La apropiación, un proceso entre muchos que ocurren en ámbitos escolares: memoria, conocimiento y utopía. Anuario de la Sociedad Mexicana de Historia de la Educación, Barcelona, n. 1, p. 28-38, enero 2004/mayo 2005.

ROCKWELL, Elsie. La experiencia etnográfica: historia y cultura en los procesos educativos. Buenos Aires: Paidós, 2009.

ROCKWELL, Elsie. Reflexiones sobre el proceso etnográfico, 1922-1985. México, DF: Departamento de Investigaciones Educativas, 1987.

SIMMEL, Georg. Sobre la individualidad y las formas sociales: escritos escogidos. Buenos Aires: Universidad Nacional de Quilmes, 2002.

TAPIA, Guillermo. Estudiantes en la transición rural-urbana del Bajío: los significados del bachillerato y del trabajo. 2015. 390 p. Tesis (Doctorado en Ciencias en Investigaciones Educativas) - Departamento de Investigaciones Educativas. Centro de Investigación y de Estudios Avanzados del Instituto Politécnico Nacional - DIE-CINVESTAV, México, DF, 2015.

TAYLOR, Charles. Fuentes del yo: la construcción de la identidad moderna. Buenos Aires: Paidós, 1996.

URTEAGA, Maritza. La construcción juvenil de la realidad. Jóvenes mexicanos y contemporáneos. 2007. 357 p. Tesis (Doctorado en Ciencias Antropológicas) - Universidad Autónoma Metropolitana, México, DF, 2007.

VEGA, Ana. Las conversaciones de los jóvenes estudiantes en el transporte público. 2013. 172 p. México DF: Tesis (Maestría en Ciencias en Investigaciones Educativas) - Departamento de Investigaciones Educativas. Centro de Investigación y de Estudios Avanzados del Instituto Politécnico Nacional - DIE-CINVESTAV, México, DF, 2013.

VELASCO, Honorio; DíAZ, Ángel. La lógica de la investigación etnográfica: un modelo de trabajo para etnógrafos de la escuela. Madrid: Trotta, 2007. 
WEISS, Eduardo. Diagnóstico de las prácticas y procesos curriculares en los bachilleratos integrales comunitarios. In: México. Secretaría de Educación Púbica. Coordinación General de Educación Intercultural y Bilingüe. Reporte de evaluación para la Coordinación General de Educación Intercultural y Bilingüe. México, DF: SEP, 2006.

WEISS, Eduardo. El abandono escolar en la educación media superior: informe de investigación para la Dirección General de Investigación Estratégica Instituto Belisario Domínguez. México, DF: Senado de la República, 2014.

WEISS, Eduardo. Hermenéutica crítica: una reflexión metodológica, sociológica y epistemológica. Paideia: Revista de la UPN, León, v. 1, n. 1, p. 7-15, jul./dic. 2005.

WEISS, Eduardo. Los estudiantes como jóvenes: el proceso de subjetivación. Perfiles Educativos, México, DF, v. 34, n. 135, p. 134-148, enero/mar. 2012b.

WEISS, Eduardo (Coord.). Jóvenes y bachillerato. México: Asociación Nacional de Universidades e Instituciones de Educación Superior, 2012a.

WEISS, Eduardo et al. Jóvenes y bachillerato en México: el proceso de subjetivación, el encuentro con los otros y la reflexividad. Propuesta Educativa, Buenos Aires, v. 18, n. 32, p. 85-104, nov. 2009.

Recibido en: 15.01.2015.

Aprobado en: 30.03.2015.

Eduardo Weiss es doctor en ciencias sociales, Universidad de Erlangen en Alemania, investigador del Departamento de Investigaciones Educativas del Centro de Investigación y de Estudios Avanzados del Instituto Politécnico Nacional (CINVESTAV) desde 1979, miembro del Consejo Mexicano de Investigación Educativa y de la Academia Mexicana de Ciencias. 